\title{
Transient analysis of reactant gas transport and performance of PEM fuel cells
}

\author{
Wei-Mon Yan ${ }^{\mathrm{a}, *}$, Chyi-Yeou Soong ${ }^{\mathrm{b}}$, Falin Chen ${ }^{\mathrm{c}}, \mathrm{Hsin}-\mathrm{Sen} \mathrm{Chu}^{\mathrm{d}}$ \\ a Department of Mechatronic Engineering, Huafan University, Shih-Ting, Taipei 223, Taiwan, ROC \\ ${ }^{\mathrm{b}}$ Department of Aerospace and System Engineering, Feng Chia University, Seatwen, Taichung 407, Taiwan, ROC \\ ${ }^{\mathrm{c}}$ Institute of Applied Mechanics, National Taiwan University, Taipei 106, Taiwan, ROC \\ ${ }^{\mathrm{d}}$ Department of Mechanical Engineering, National Chiao Tung University, Hsin-Chu 300, Taiwan, ROC
}

Received 28 May 2004; received in revised form 4 November 2004; accepted 9 November 2004

Available online 2 February 2005

\begin{abstract}
The dynamic performance of PEM fuel cells is one of the most important criteria in the design of fuel cells with application to mobile systems. To study this issue, we extend our previous steady model of gas reactant transport to an unsteady one and employ it to examine the transient transport characteristics and the system performance of the PEM fuel cells. With the assumption of the two-dimensional mass transport in the cathode side of PEMFC, the effects of the channel width fraction, $\lambda=\ell_{\mathrm{c}} / \ell_{\mathrm{b}}$, the porosity of the gas diffuser layer, $\varepsilon_{1}$, and the surface overpotential of the catalyst layer, $\eta$, on the transient characteristics of the resultant current density and mass transport are focused in this work. It is disclosed that an increase in $\lambda$, or $\eta$ may lead to a faster dynamic response for the fuel cell when the PEM fuel cell system is started up. Results of a typical case demonstrate that, although the dynamic response time may be as long as $10 \mathrm{~s}$ due to the mass transport lag, the fuel cell system needs only less than $0.4 \mathrm{~s}$ to reach the $90 \%$ response.
\end{abstract}

(C) 2005 Elsevier B.V. All rights reserved.

Keywords: PEM fuel cell; Transient response; Gas transport; Channel width fraction; Porosity

\section{Introduction}

Proton exchange membrane (PEM) fuel cells have been recognized as a promising candidate of power generators for both stationary and automotive applications due to their merits of low operating temperature, high efficiency and extremely low pollutant emission. In applications of PEM fuel cells to mobile systems, the dynamic response to the start-up of the system is important. In addition, the time interval from the start of operation to the steady state is one of the significant performance characteristics of the system. To attack the transient behaviors of the PEM fuel cell, the time interval and other potential time constants, the unsteady reactant gas transport in the PEM fuel cell shall be considered. The dynamic

\footnotetext{
* Corresponding author. Tel.: +886 22663 2102; fax: +8862 26631119.

E-mail address: wmyan@huafan.hfu.edu.tw (W.-M. Yan).
}

response of the fuel cell depends strongly on the operating conditions, transport phenomena in the cells, electrochemical reaction kinetics, mechanical design and manufacturing process, etc. Among these, the reactant gas transport in the flow distributor and the gas diffusion layer (GDL) before occurrence of the chemical reaction is one of the crucial points.

The GDL morphology, geometry and porosity, as well as the flow channel arrangement, e.g., parallel, serpentine or interdigited channel, have significant influences on optimization of the cell performance. In the past years, studies related to the above issues have been performed. Bernardi and Verbrugge [1] developed a one-dimensional model to examine the proton transport behaviors in the membrane. Their results indicated that if the membrane is maintained at fully saturated condition, the resistance then becomes significant at the current densities greater than $200 \mathrm{~mA} \mathrm{~cm}^{-2}$. Okada and colleagues [2-7] studied water transport with and without im- 


$\begin{array}{ll}\text { Nomenclature } \\ D & \text { Mass diffusivity, } \mathrm{m}^{2} \mathrm{~s}^{-1} \\ F & \text { Faraday constant, } 96487 \mathrm{C} \mathrm{mol}^{-1} \\ I & \text { current density, } \mathrm{A} \mathrm{m}^{-2} \\ I_{0} & \text { maximum current density at } X=0, \mathrm{~A} \mathrm{~m}^{-2} \\ j & \text { transfer current density, } \mathrm{A} \mathrm{m}^{-2} \\ \ell_{\mathrm{b}} & \text { base width of the flow distributor, } \mathrm{m} \\ \ell_{\mathrm{c}} & \text { gas channel width, } \mathrm{m} \\ \ell_{\mathrm{s}} & \text { shoulder width, } \mathrm{m} \\ M_{\mathrm{O}_{2}} & \text { molecular mass of oxygen, } 32.0 \mathrm{~kg} \mathrm{kmol}^{-1} \\ N & \text { number of flow channels } \\ n & \text { number of electrons in transfer current density } \\ & \text { relation } \\ P & \text { pressure, Pa } \\ R & \text { universal gas constant, } 8.314 \mathrm{~J} \mathrm{~mol} \mathrm{~m}^{-1} \mathrm{~K}^{-1} \\ T & \text { temperature, } \mathrm{K} \\ t & \text { time, } \mathrm{s} \\ s_{\mathrm{c}} & \text { thickness of catalyst-layer, } \mathrm{m} \\ s_{\mathrm{d}} & \text { thickness of diffusion layer, } \mathrm{m} \\ s_{\mathrm{m}} & \text { thickness of membrane layer, } \mathrm{m} \\ V & \text { cell voltage } \\ w & \text { oxygen mass fraction } \\ w_{\mathrm{o}} & \text { oxygen mass fraction at inlet }\end{array}$

\section{Greek letters}

$\begin{array}{ll}\alpha & \text { transfer coefficient for the reaction } \\ \varepsilon_{1} & \text { porosity of diffusion layer } \\ \varepsilon_{2} & \text { porosity of catalyst layer } \\ \varepsilon_{\mathrm{i}} & \text { volume fraction of ionomer in the catalyst layer } \\ \eta & \text { surface overpotential, } \mathrm{V} \\ \kappa & \text { reaction rate constant, } \mathrm{mol} \mathrm{m}^{-3} \mathrm{~s}^{-1} \\ \lambda & \text { channel width fraction, } \ell_{\mathrm{c}} / \ell_{\mathrm{b}} \\ \lambda_{\mathrm{w}} & \text { membrane water content, } \mathrm{kmol} \\ & \mathrm{H}_{2} \mathrm{O} / \mathrm{kmol} \mathrm{SO}_{3}{ }^{-} \\ \rho & \text { density of oxygen, } \mathrm{kg} \mathrm{m}^{-3} \\ \sigma & \text { ionic conductivity of the ionomer, } \Omega^{-1} \mathrm{~m}^{-1} \\ \tau_{1} & \text { tortuosity of diffusion layer } \\ \tau_{2} & \text { tortuosity of catalyst layer } \\ \tau_{\mathrm{i}} & \text { tortuosity of the ionomer in catalyst layer } \\ \phi & \text { ionomer phase potential, } \mathrm{V}\end{array}$

purity ions in membranes of PEM fuel cells. Gurau et al. [8] proposed a one-dimensional model to examine the characteristics of the mass transport of reactant gas in a half-cell, in which the effects of the porosity and the tortuosity of GDL and the catalyst layer were investigated.

For more details of the steady-state flow distribution and gas diffusion, a number of studies on two- and threedimensional transport phenomena in PEM fuel cells have been carried out. Dutta et al. [9] used the software package to analyze the distributions of gas density and velocity in flow channel and GDL. Nguyen and coworkers [10,11] developed a two-dimension model to examine the water and thermal management of PEM fuel cells of interdigitated flow channel. They found that the interdigitated gas distributors can reduce mass-transport overpotential and improve the flooding phenomena in the cathode side. In addition, it is found that the cell performance can be enhanced by decreasing the electrode thickness or increasing the number of the gas channels. To obtain an optimization of the flow field and to improve the fuel cell performance, Kee et al. [12] employed the Navier-Stokes equations as the flow model and the Michaelis-Menten type two-step kinetics as the gas reaction model at the anode to explore the effects of flow distributors. For flow distribution in channel networks of typical planar fuel cell layers and stacks, Hontabon et al. [13] proposed a generalized steady-state computational model, in which only mass and momentum transfer are considered. Um et al. [14] studied two-dimensional transport phenomenon of the PEM fuel cells numerically. The results showed that the hydrogen dilution deteriorates the cell performance. Most recently, Yan et al. [15] developed a two-dimensional model to investigate gas reactant transport at various conditions of flow channel width ratio and GDL porosity. They disclosed that an increase in either $\lambda$ or $\varepsilon$ may lead to a better cell performance. At relatively low overpotential, better uniformity in current density distribution along the width of the cell can be attained.

The previous studies mentioned above are all concerned with the steady-state transport phenomena and the system performance of PEM fuel cells. In application of the fuel cells as power systems of automobiles, start-up time is probably a most significant performance index. However, in the past years, only a few analyses focused their attentions on the transient characteristics of PEM fuel cells. Francesco and Arato [16] investigated the transient response of an automotive power system by employing a model of fuel cell stack and a compressor. Their model and results showed that a proper range of the values of the air relative humidity and temperature must be maintained for system start-up under stable condition. Yerramalla et al. [17] developed a mathematical model for investigating the dynamic performance of a PEM fuel cell with and without assumptions for linearization. Significant differences between the linear and non-linear results were found. Without special emphasis on the transient behaviors of the fuel cell systems, Um and Wang [18] developed a three-dimensional unsteady computational fuel cell dynamics (CFCD) model for analysis of interactions between mass transport and electrochemical kinetics. In a recent investigation by our group, Chen et al. [19], we investigated the transient behavior of the water transport across the membrane of the PEM fuel cell to seek for effective control schemes so that the best dynamic performance of the fuel cell can be obtained. It is found that both a larger starting operational current density and a smaller operational current density can lead to a shorter dynamic response time, while control of the humidification of the fed fuel is the most powerful as well as the most feasible scheme. 
The objective of this work is to study the effects of the flow distributor design and the GDL morphology on the transient behaviors of reactant gas transport and dynamic cell performance of a fuel cell system subjected to a start-up operation. The dynamic response time for the system state changing from the start-up operation to the steady state will be examined. The emphasis of the analysis is placed on the influences of the channel width characterized by a fraction $\lambda=\ell_{\mathrm{c}} / \ell_{\mathrm{b}}$ (=channel width/base plate width), and the porosity of the GDL. By applying the time-dependent mass transport model developed in the present analysis, the effects of these two parameters and the overpotential on the transient characteristics of the mass transport and the dynamic response of the cell performance are explored.

\section{Theory}

\subsection{Model development}

A schematic diagram of the cross-sectional view of a halfPEM fuel cell model is shown in Fig. 1 , where $S_{\mathrm{m}}, S_{\mathrm{c}}$, and $S_{\mathrm{d}}$ denote thicknesses of membrane, catalyst layer, and GDL, respectively. The parameters $\ell_{\mathrm{c}}, \ell_{\mathrm{s}}$, and $\ell_{\mathrm{b}}=\ell_{\mathrm{c}}+\ell_{\mathrm{s}}$ stand for the widths of the channel, the shoulder and the base plate, respectively. The area ratio between flow channel and shoulder is one of the most important geometry parameters on the behaviors of the PEM fuel cells. With the assumption of the two-dimensional mass transport in the cathode side of PEMFC, the unsteady two-dimensional transport equations for the oxygen mass fraction, $w$, in diffuser layer and catalyst layer can be expressed as follows:
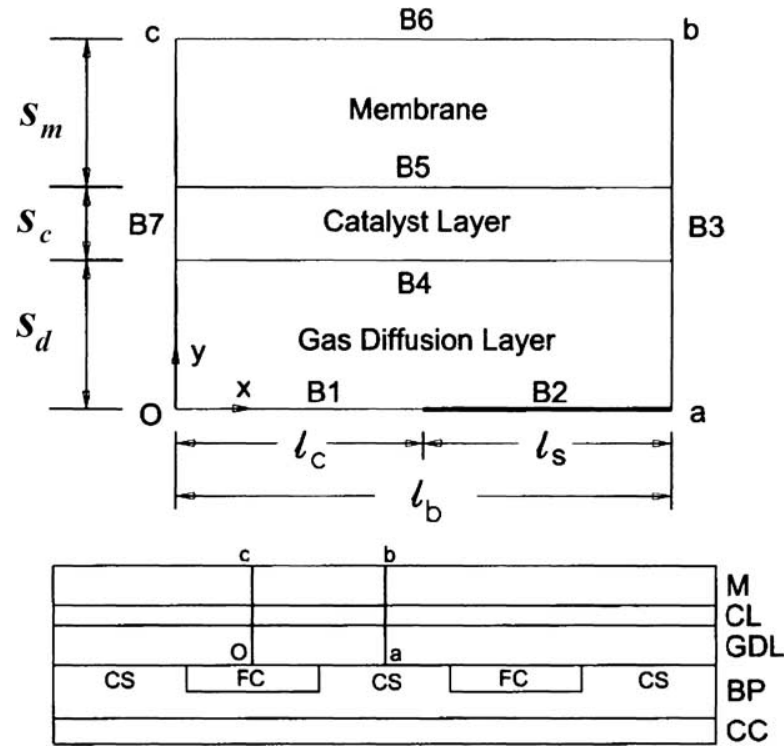

Fig. 1. Half-cell model of a PEM fuel cell. FC = flow channel; $\mathrm{CS}=$ channel shoulder; $\mathrm{CC}=$ current collector; $\mathrm{BP}=$ bipolar plate; $\mathrm{GDL}=$ gas diffusion layer; $\mathrm{CL}=$ catalyst layer; $\mathrm{M}=$ membrane; $\mathrm{B} 1 \sim \mathrm{B} 7=$ boundary numbers [15].
(A) In the diffuser layer

$$
\rho \varepsilon_{1} \frac{\partial w}{\partial t}=\frac{\partial}{\partial x}\left(\varepsilon_{1}^{\tau_{1}} \rho D \frac{\partial w}{\partial x}\right)+\frac{\partial}{\partial y}\left(\varepsilon_{1}^{\tau_{1}} \rho D \frac{\partial w}{\partial y}\right)
$$

(B) In the catalyst layer

$$
\begin{aligned}
\rho \varepsilon_{2} \frac{\partial w}{\partial t}= & \frac{\partial}{\partial x}\left(\varepsilon_{2}^{\tau_{2}} \rho D \frac{\partial w}{\partial x}\right)+\frac{\partial}{\partial y}\left(\varepsilon_{2}^{\tau_{2}} \rho D \frac{\partial w}{\partial y}\right) \\
& -\frac{j}{2 n F} M_{\mathrm{O}_{2}}
\end{aligned}
$$

where $D$ stands for the mass diffusivity of the gas, $\varepsilon$ and $\tau$ are, respectively, the porosity and tortuosity factor of the catalyst layer. While $n$ denotes the number of electrons in transfer current density relation and $F$ is the Faraday constant. In Eq. (2), $j$ represents the cathode transfer current density and can be formulated by the following equation under the assumption that the oxygen reduction reaction is irreversible, first-order in oxygen and that electroneutrality holds in any representative elementary volume,

$j=n F \kappa w \exp \left(\frac{\alpha n F}{R T}\right)$

Substituting Eq. (3) into (2) yields

$$
\begin{aligned}
\rho \varepsilon_{2} \frac{\partial w}{\partial t}= & \frac{\partial}{\partial x}\left(\varepsilon_{2}^{\tau_{2}} \rho D \frac{\partial w}{\partial x}\right)+\frac{\partial}{\partial y}\left(\varepsilon_{2}^{\tau_{2}} \rho D \frac{\partial w}{\partial y}\right) \\
& -\frac{\kappa M_{\mathrm{O}_{2}}}{2} \exp \left(\frac{\alpha n F}{R T} \eta\right) w
\end{aligned}
$$

In the membrane and the catalyst layer, the phase potential equations can be expressed as follows:

(A) In the membrane

$$
\frac{\partial}{\partial x}\left(\sigma \frac{\partial \phi}{\partial x}\right)+\frac{\partial}{\partial y}\left(\sigma \frac{\partial \phi}{\partial y}\right)=0
$$

(B) In the catalyst layer

$$
\frac{\partial}{\partial x}\left(\varepsilon_{\mathrm{i}}^{\tau_{\mathrm{i}}} \sigma \frac{\partial \phi}{\partial x}\right)+\frac{\partial}{\partial y}\left(\varepsilon_{\mathrm{i}}^{\tau_{\mathrm{i}}} \sigma \frac{\partial \phi}{\partial y}\right)=j
$$

Due to the presence of transfer current density in the catalyst layer, there exists a source term in Eq. (6). In addition, $\varepsilon_{i}$ and $\tau_{\mathrm{i}}$ stands for the volume fraction and the tortuosity factor of the ionomer in the catalyst layer, and $\sigma$ for the membrane ionic conductivity and is evaluated by

$$
\sigma=\left(0.5139 \lambda_{\mathrm{w}}-0.326\right) \exp \left[1268\left(\frac{1}{303}-\frac{1}{T}\right) \times 10^{-2}\right]
$$

\subsection{Initial and boundary conditions}

In this work, the fuel cell system is assumed to be stationary initially $(t<0)$. At time $t=0$, the fuel cell system is subjected to a start-up operation, thereafter, the system shows 
a transient response until the steady state is reached. Therefore, the initial condition about the mass fraction $w$ is zero. As for the boundary conditions with the boundaries numbered B1 to B7 in Fig. 1, they can be described as follows. The boundary conditions for the oxygen mass fraction $w$ are,

$w=w_{0} \quad$ at boundary $\mathrm{B} 1$;

$\frac{\partial w}{\partial y}=0 \quad$ at boundaries B2 and B5;

$\frac{\partial w}{\partial x}=0 \quad$ at boundaries B3 and B7;

$w_{-}=w_{+}, \quad \varepsilon_{1}^{\tau_{1}} D \frac{\partial w}{\partial y}=\varepsilon_{2}^{\tau_{2}} D \frac{\partial w}{\partial y} \quad$ at boundary B4.

The boundary conditions for phase potential $\phi$ are,

$\frac{\partial \phi}{\partial y}=0 \quad$ at boundary B4;

$\frac{\partial \phi}{\partial x}=0 \quad$ at boundaries B3 and B7;

$\phi_{-}=\phi_{+},\left.\quad \varepsilon_{\mathrm{i}}^{\tau_{\mathrm{i}}} \frac{\partial \phi}{\partial y}\right|_{-}=\left.\frac{\partial \phi}{\partial y}\right|_{+} \quad$ at boundary B5;

$\phi=0 \quad$ at boundary B6.

\subsection{Evaluation of cell potential}

The cell potential $V$ is evaluated by subtracting surface overpotential $\eta$ and the average phase potential difference $\Delta \phi$ from the ideal cell potential $V_{0 c}$, viz.,

$V=V_{0 \mathrm{c}}-\eta-\Delta \phi$

and the value of $\Delta \phi$ can be evaluated by the following expression,

$\Delta \phi=\frac{\int_{0}^{\ell_{\mathrm{b}}}\left(\phi_{6}-\phi_{4}\right) \mathrm{d} x}{\ell_{\mathrm{b}}}$

in which $\phi_{4}$ and $\phi_{6}$, respectively, denote the phase potentials at the boundaries B4 and B6.

\subsection{Evaluation of time constant}

In the study of the transient behaviors of the PEM fuel cells, the time interval and the time constant are important when the system is subjected to a start-up operation. In this work, the time constant is defined as the time needed for the value of current density reaching $90 \%$ of that at the steady state.

\section{Numerical method}

The above set of equations for time-dependent transport in PEM fuel cells have to be solved numerically. In the present study, we adopt a fully implicit finite difference method in which the time derivative term is approximated by the backward difference method and the space derivative terms by the central difference. With the coupled finite-difference equations, the linear algebraic equations are solved by the Thomas algorithm [20] with iterations. In this work, uniformly distributed grids having 201 and 221 points are used in the $x$ and $y$ directions, respectively. To obtain enhanced accuracy in the numerical computations, grid independence is tested in the separate numerical runs. It is found that the deviations between grid points of $201 \times 201$ and $401 \times 401$ with first time interval 0.001 are less than $2 \%$. Accordingly, the computations on the grid system of $201 \times 201$ points in the $x$ and $y$ directions with first time interval 0.001 are sufficient to understand the transient behaviors of the reactant gas transport in the PEM fuel cells. To further check the adequacy of the numerical scheme, the limiting case of steady one-dimensional model is first evaluated. The predicted results agree well with those of Gurau et al. [8]. Through the above tests, it confirms that the presented model and numerical method both are appropriate for the present problem.

\section{Results and discussion}

The values of the parameters considered in the present study are listed in Table 1 . The numerical results are presented to discuss the effects of various parameters. The geometry is characterized by a parameter named channel width fraction, $\lambda \equiv \ell_{\mathrm{c}} / \ell_{\mathrm{b}}$, and three values, $\lambda=0.25,0.5$, and 0.75 , are considered. In addition, the effects of the GDL morphol-

Table 1

Parameters used in the present analysis

\begin{tabular}{|c|c|c|}
\hline Parameters & Notation & Value \\
\hline Temperature, $\mathrm{K}$ & $T$ & 333 \\
\hline Pressure, $\mathrm{Pa}$ & $P$ & 303975 \\
\hline Reaction rate constant, $\mathrm{mol} \mathrm{m}^{-3} \mathrm{~s}^{-1}$ & $\kappa$ & $1.5 \times 10^{-5}$ \\
\hline Oxygen mass fraction at inlet & $w_{0}$ & 0.17 \\
\hline Gas channel width, $m$ & $\ell_{\mathrm{c}}$ & $5 \times 10^{-4}$ \\
\hline Channel shoulder width, $\mathrm{m}$ & $\ell_{\mathrm{s}}$ & $5 \times 10^{-4}$ \\
\hline Thickness of diffusion layer, $m$ & $s_{\mathrm{d}}$ & $5 \times 10^{-4}$ \\
\hline Thickness of catalyst layer, $\mathrm{m}$ & $s_{\mathrm{c}}$ & $5 \times 10^{-5}$ \\
\hline Thickness of membrane, $\mathrm{m}$ & $s_{\mathrm{m}}$ & $2.0 \times 10^{-4}$ \\
\hline Porosity of GDL & $\varepsilon_{1}$ & 0.8 \\
\hline Tortuosity of GDL & $\tau_{1}$ & 1.5 \\
\hline Porosity of catalyst layer & $\varepsilon_{2}$ & 0.3 \\
\hline Tortuosity of catalyst layer & $\tau_{2}$ & 1.5 \\
\hline Volume fraction of ionomer in CL & $\varepsilon_{\mathrm{I}}$ & 0.3 \\
\hline Ionomer tortuosity in CL & $\tau_{\mathrm{I}}$ & 1.5 \\
\hline Faraday constant, $\mathrm{c} \mathrm{mol}^{-1}$ & $F$ & 96487 \\
\hline $\begin{array}{l}\text { Universal gas constant, } \mathrm{kJ} \mathrm{kmol}^{-1} \mathrm{~K}^{-1} \\
\text { or } \mathrm{J} \mathrm{mol}^{-1} \mathrm{~K}^{-1}\end{array}$ & $R$ & 8.314 \\
\hline Molecular mass of oxygen, $\mathrm{kg} \mathrm{kmol}^{-1}$ & $M$ & 32.0 \\
\hline $\begin{array}{l}\text { Number of electrons in relation } \\
\text { participating }\end{array}$ & $n$ & 2 \\
\hline Current density, $\mathrm{A} \mathrm{m}^{-2}$ & $I$ & \\
\hline Surface overpotential, V & $\eta$ & \\
\hline Ionomer phase potential, $\mathrm{V}$ & $\phi$ & \\
\hline
\end{tabular}



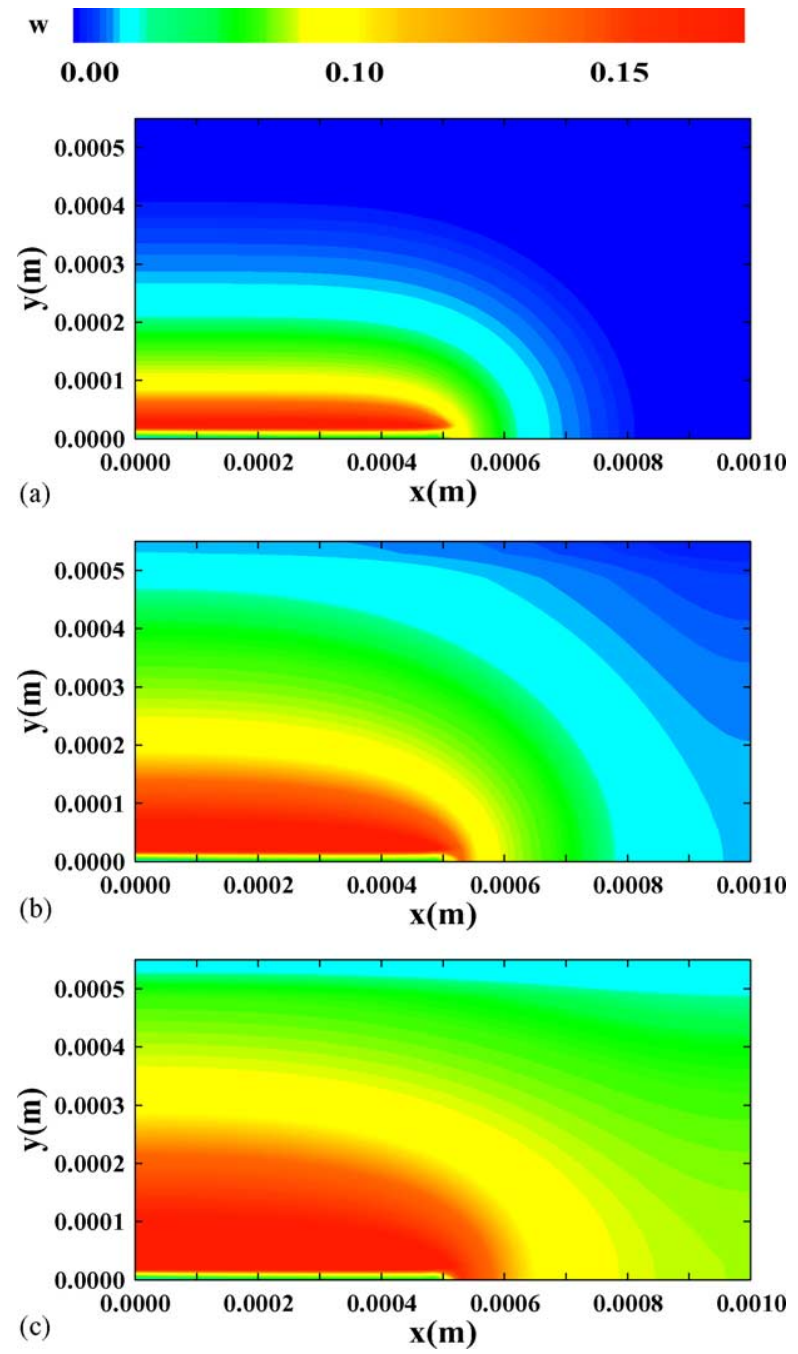

Fig. 2. Oxygen mass fraction contours in diffusion and catalyst layers with $\lambda=0.50$ and $\eta=0.2$ at different transients: (a) $t=0.01$; (b) $t=0.05$; (c) $t=8.35$.

ogy are explored by considering the values of the GDL porosity in the range of $0.3 \leq \varepsilon \leq 0.6$.

Fig. 2 presents the contours of the oxygen mass fraction in the GDL $\left(0 \leq y \leq 5 \times 10^{-4}\right)$ and catalyst layer $\left(5 \times 10^{-4} \leq y \leq 5.5 \times 10^{-4}\right)$ with $\varepsilon_{1}=0.5, \eta=0.2$ and $\lambda=0.5$ at time instants $t=0.01,0.05$ and 8.35. The right half of the $x$-axis in each contour corresponds the axial location of the shoulder (or rib) of the channel. An overall inspection of Fig. 2 discloses that in the region corresponding to the channel central region (left portion of the contour), the relatively higher oxygen mass fraction appears and, then, the oxygen diffuses towards the porous material covering the rib where there is no oxygen source on the interface $y=0$. In the early initial stage, see Fig. 2(a), only a higher oxygen mass fraction presents in the region near the inlet of channel central region only. This is because the oxygen fuel gas does not have sufficient time to penetrate in depth when the system is just started up. As time elapses, however, the oxygen diffuses to the regions far from the inlet. Finally, the system researches
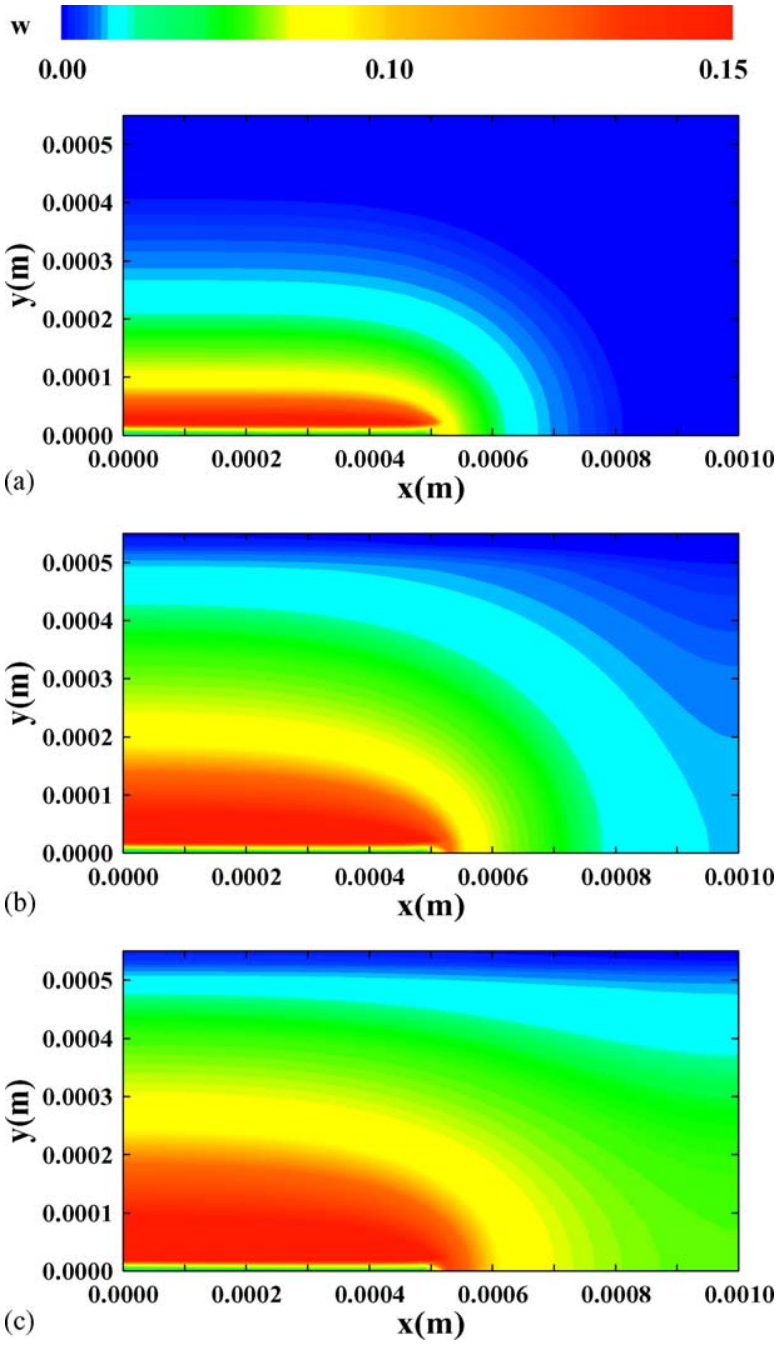

Fig. 3. Oxygen mass fraction contours in diffusion and catalyst layers with $\lambda=0.5$ and $\eta=0.225$ at different transients: (a) $t=0.01$; (b) $t=0.05$; (c) $t=4.215$.

at a steady state after experiences a time period of transient variation.

In this work, the surface overpotential $\eta$ represents the voltage loss due to chemical reaction within the catalyst layer. Therefore, a higher surface overpotential means a fuel cell with a faster surface reaction rate. To explore effects of the surface overpotential on the reactant gas transport, the contours of the oxygen mass fraction in the gas diffuser layer and catalyst layer of $\varepsilon_{1}=0.5, \eta=0.225$ and $\lambda=0.75$ at different instants are shown in Fig. 3. Comparison of Figs. 2 and 3 discloses that a smaller oxygen concentration is noted for a system with a high surface overpotential $\eta$. This is resulted from the fact that a PEM fuel cell with a higher surface overpotential represents a fuel cell with a faster surface reaction rate. This explains why the dynamic response is faster in the case with a higher $\eta$.

To examine the effects of the channel width fraction $\lambda$ on the reactant gas transport, the temporal variation of the oxygen mass fraction in the gas diffuser layer and catalyst 

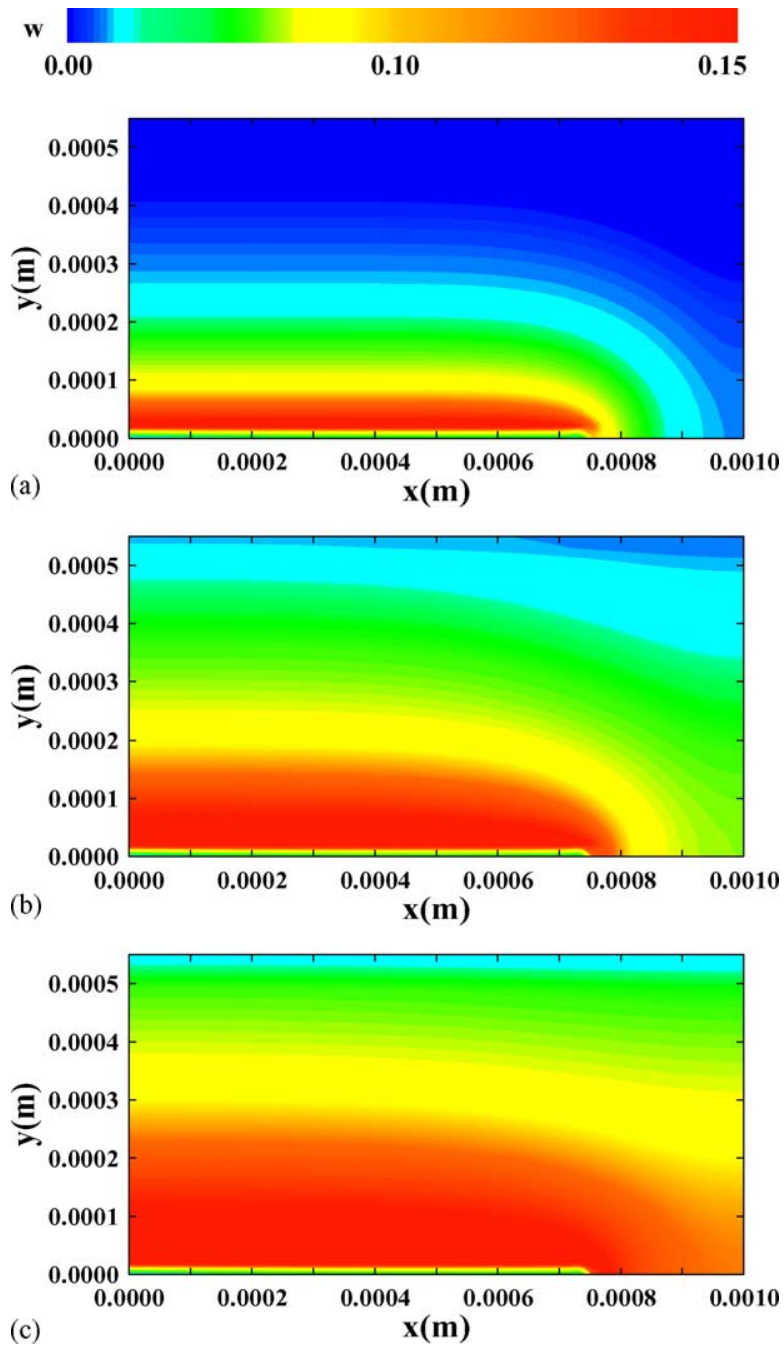

Fig. 4. Oxygen mass fraction contours in diffusion and catalyst layers with $\lambda=0.75$ and $\eta=0.2$ at different transients: (a) $t=0.01$; (b) $t=0.05$; (c) $t=2.184$.

layer of $\varepsilon_{1}=0.5, \eta=0.2$ and $\lambda=0.75$ are shown in Fig. 4 . Comparing with the results in Fig. 2, it is shown that, in the case with a larger channel width fraction, the distribution of oxygen is more uniform. This means that the concentration gradient of the oxygen is more noticeable in the case of a smaller channel width fraction, i.e. $\lambda=0.5$. Additionally, the time needed for the system to reach the steady state is longer for a smaller $\lambda$. This can be made plausible by noting the fact that for a narrow channel, the effective inlet region is smaller. In this situation, it takes a longer penetration time to diffuse oxygen to the steady state when the system is started up.

Fig. 5 presents the local oxygen fraction distributions along the interface between gas diffuser layer and catalyst layer, i.e. boundary B4 in Fig. 1, under various conditions. It is noteworthy that in the early state, e.g. $t=0.01$, the oxygen fraction is almost zero. This means that the oxygen does not penetrate to the interface at this short duration. Later, the oxygen fraction increases with time till steady state is reached. With the periodic conditions at left and right sides, i.e. bound-

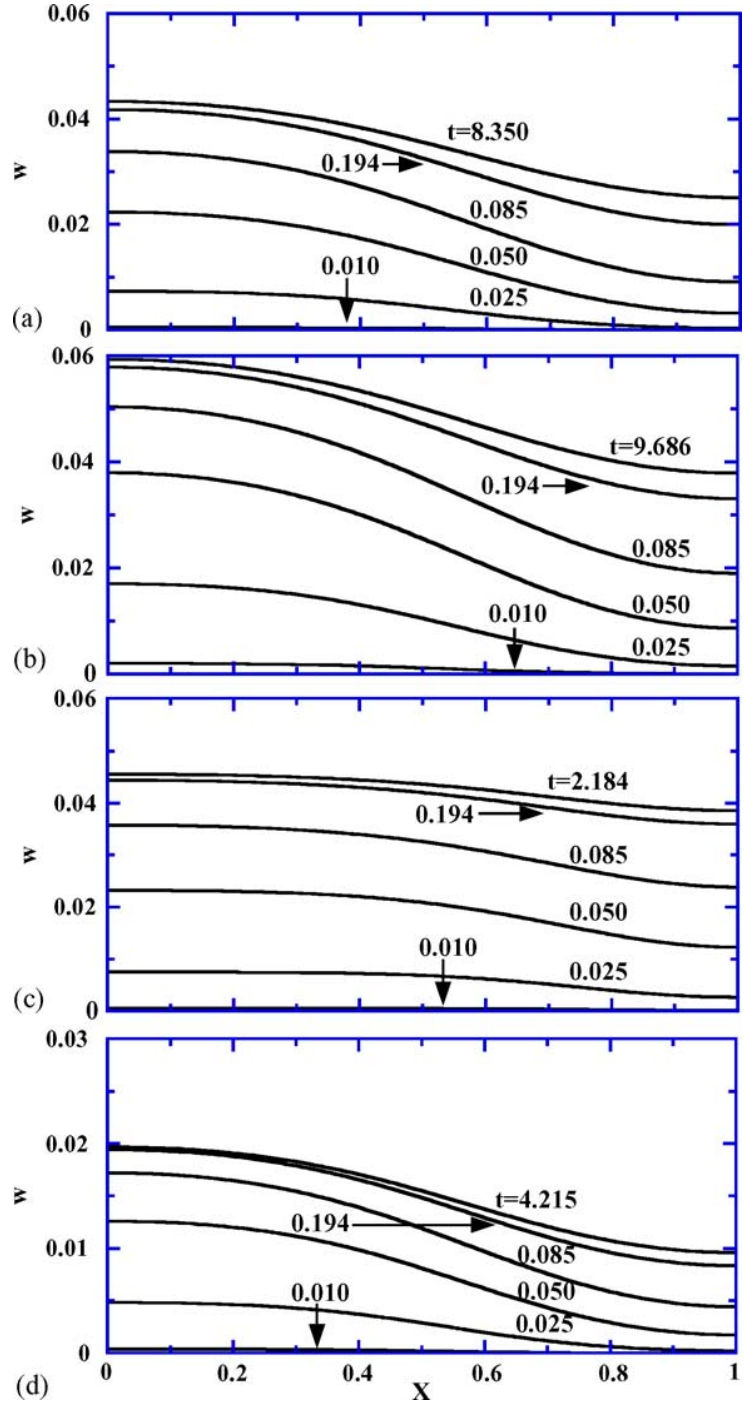

Fig. 5. Transient variations of oxygen mass fraction along interface between the gas diffuser layer and catalyst layer: (a) $\varepsilon_{1}=0.5, \lambda=0.5$ and $\eta=0.2$; (b) $\varepsilon_{1}=0.6, \lambda=0.5$ and $\eta=0.2$; (c) $\varepsilon_{1}=0.5, \lambda=0.75$ and $\eta=0.2$; (d) $\varepsilon_{1}=0.5$, $\lambda=0.5$ and $\eta=0.225$.

aries 3 and 7, the distributions of the mass fraction at the left sides (i.e., $x<0.5$ ) are higher than that at right sides. A careful examination of Fig. 5(a) indicates that as the time is about 0.194 , the mass fraction is very close to that at steady state $(t=8.35)$. It is found from a separate numerical run that at $t=1.0$, the mass fraction almost collapses with that at steady state. This implies that, after the PEM fuel cell system is started up, it arrives at the steady very quickly. Comparison of the corresponding curves of $t=0.01 \mathrm{~s} \mathrm{in} \mathrm{Fig.} \mathrm{5(a)} \mathrm{and} \mathrm{(b)}$ discloses that the dynamic response to the power surge of the PEM fuel cell is faster in the initial stage for a system with a higher GDL porosity. This is owing to the fact that a high GDL porosity enhances the diffusion transport of the reactant gas through the porous layers and, in turn, increases the local values of the oxygen mass fraction. However, the time needed for the system to reach the steady state is longer for a PEM fuel cell with a higher GDL porosity. As explained in Fig. 3, 
the distributions of the mass fraction along the interface between the GDL and catalyst layer are relatively uniform for a larger channel width fraction, see Fig. 5(a) and (c). Besides, a shorter dynamic response time is experienced for a PEM fuel cell with a larger channel width fraction. Fig. 5(d) shows the transient variations of the mass fraction with $\varepsilon_{1}=0.5$, $\eta=0.225$ and $\lambda=0.5$. Relative to Fig. 5(a), it is observed that an increase in the surface overpotential $\eta$ results in a decrease in the mass fraction. This phenomenon is attributed to the fact that a PEM fuel cell with a higher surface overpotential represents a fuel cell with a faster surface reaction rate. Therefore, the mass fraction along the interface of the catalyst layer is lower. In addition, the response time is shorter for a PEM fuel cell with a higher $\eta$.

In Fig. 6, the unsteady phase potential distributions along the interface between the catalyst layer and the membrane at the conditions corresponding to that in Fig. 5 are presented. An overall inspection on Fig. 6 discloses that, in the early transient, the value of the phase potential is almost zero, i.e., the value of initial condition. But as the time elapses, the phase potential decreases until the system becomes steady. As

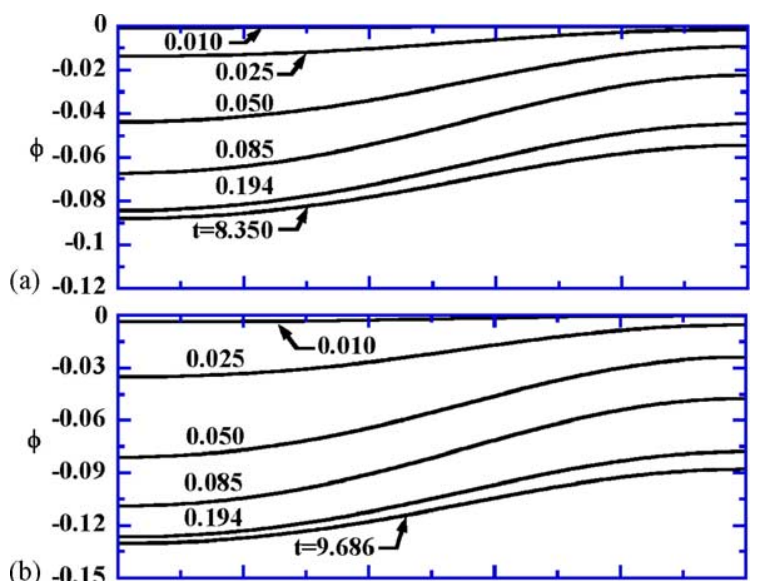

(b)

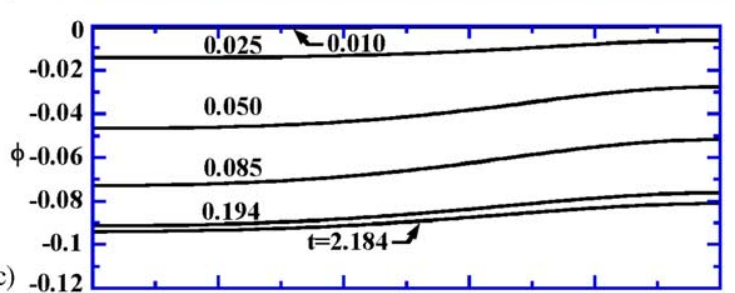

(d)

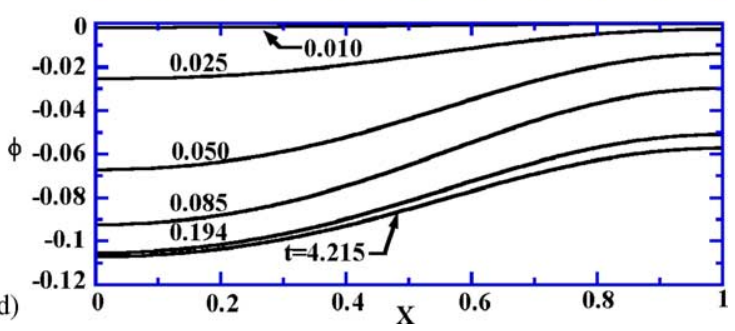

Fig. 6. Transient variations of phase potential along interface between the gas diffuser layer and catalyst layer: (a) $\varepsilon_{1}=0.5, \lambda=0.5$ and $\eta=0.2$; (b) $\varepsilon_{1}=0.6, \lambda=0.5$ and $\eta=0.2$; (c) $\varepsilon_{1}=0.5, \lambda=0.75$ and $\eta=0.2$; (d) $\varepsilon_{1}=0.5$, $\lambda=0.5$ and $\eta=0.225$.
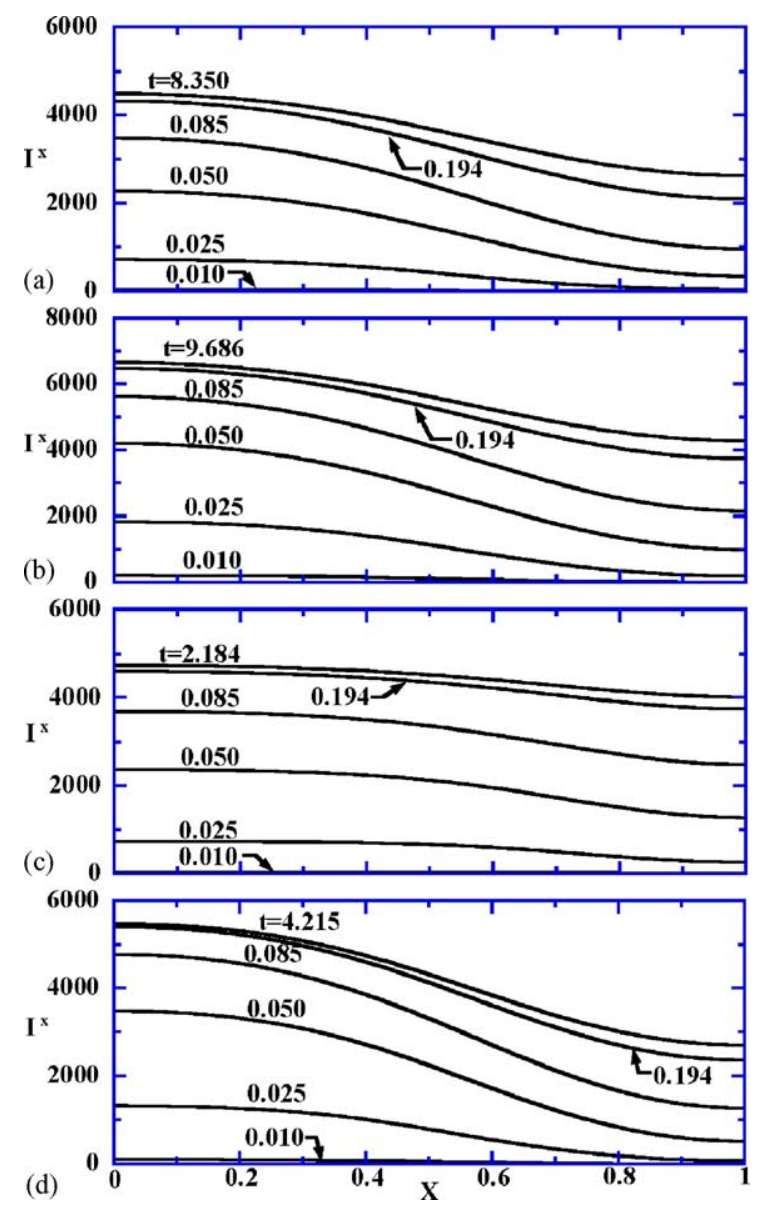

Fig. 7. Transient variations of local current density along interface between the gas diffuser layer and catalyst layer: (a) $\varepsilon_{1}=0.5, \lambda=0.5$ and $\eta=0.2$; (b) $\varepsilon_{1}=0.6, \lambda=0.5$ and $\eta=0.2$; (c) $\varepsilon_{1}=0.5, \lambda=0.75$ and $\eta=0.2$; (d) $\varepsilon_{1}=0.5$, $\lambda=0.5$ and $\eta=0.225$.

the interpretation for the results in Fig. 5, a shorter dynamic response time is noted for a PEM fuel cell with a higher GDL porosity, channel width fraction and surface overpotential. In fact, with the results of the phase potential distributions, the average phase potential difference and the voltage can then be obtained.

The distributions of the local current density along the interface at various instants with the same conditions in Figs. 5 and 6 are shown in Fig. 7. Slow response of the system in the early initial transient is again demonstrated by the very low current density. With the time going, the oxygen mass fraction penetrates into the catalyst layer from the inlet. Thereafter, the oxygen reacts and produces the significant amount of current. Additionally, a higher current density is found for a PEM fuel cell with a higher GDL porosity, channel width fraction and surface overpotential. Since the increase in GDL porosity enhances the diffusion transport of the reactant gas through the porous layers and, in turn, increases the local values of the current density with a specified overpotential (by comparing Fig. 7(a) and (b)). However, this high transport rate of the reactant gas in the across-the-cell direction may result in a relatively non-uniformity in $x$-distribution of the 

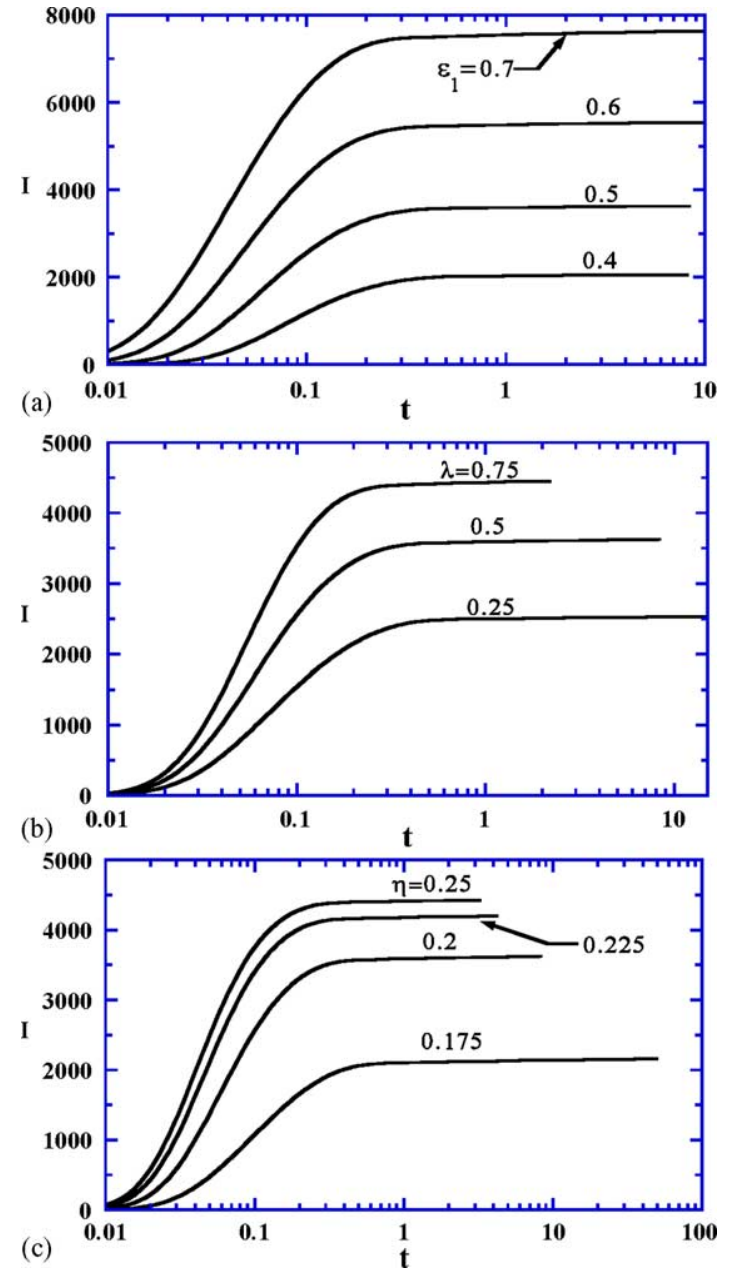

Fig. 8. Transient variations of current density: (a) $\lambda=0.5$ and $\eta=0.2$; (b) $\varepsilon_{1}=0.5$ and $\eta=0.2$; (c) $\varepsilon_{1}=0.5$ and $\lambda=0.5$.

current density as a penalty. A careful comparison of Fig. 7(a) and (d) indicates that the distribution of the current density in $x$-direction is relatively uniform at low overpotentials. At a high overpotential, the surface reaction rate becomes fast and the relatively weak diffusion leads to a non-uniformity in the current density distribution.

In the study of the transient behaviors of reactant gas transport in PEM fuel cell, the dynamic response time for the PEM fuel cell subjected to a power surge is important under various conditions. Therefore, the temporal variations of the current density and voltage for different conditions are presented in Figs. 8 and 9, respectively. It is disclosed that when the PEM fuel cell is subjected to a power surge from the stationary condition, the PEM fuel cell responds quickly to the change. Although, the full time duration to the steady state is much longer than $1 \mathrm{~s}$, but the PEM fuel cell almost changes to the $99 \%$ value at steady within $1 \mathrm{~s}$. In addition, a drastic response occurs at the instant of about $0.1 \mathrm{~s}$. It is found from the separate numerical data that at the instant less than $0.001 \mathrm{~s}$, the PEM fuel cell does not respond to the change in the operating condition. This means that the PEM fuel cell is still stationary with almost null current density and
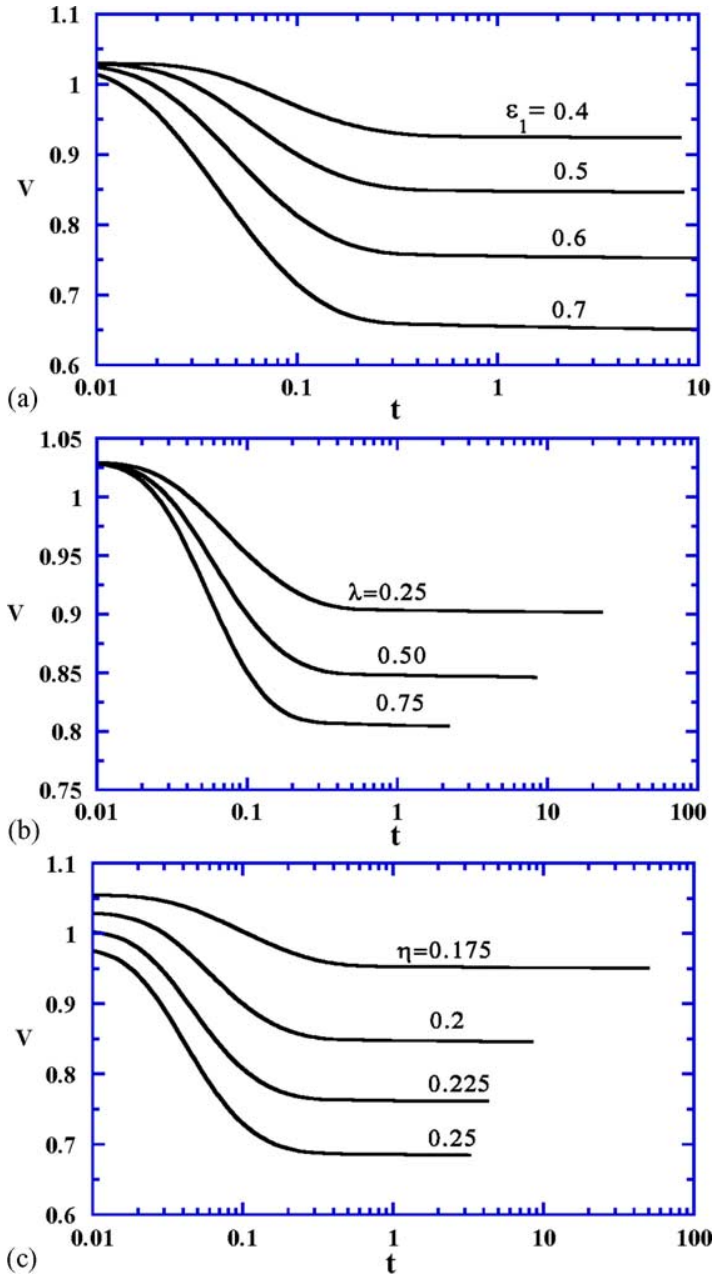

Fig. 9. Transient variations of voltage: (a) $\lambda=0.5$ and $\eta=0.2$; (b) $\varepsilon_{1}=0.5$ and $\eta=0.2 ;$ (c) $\varepsilon_{1}=0.5$ and $\lambda=0.5$.

voltage. In Figs. 8(a) and 9(a), the effects of the GDL porosity on the current density and dynamic response time can be observed.

In the study of the transient response of a PEM fuel cell system, the time constant is one of significant parameters for evaluation of the system performance, especially for the

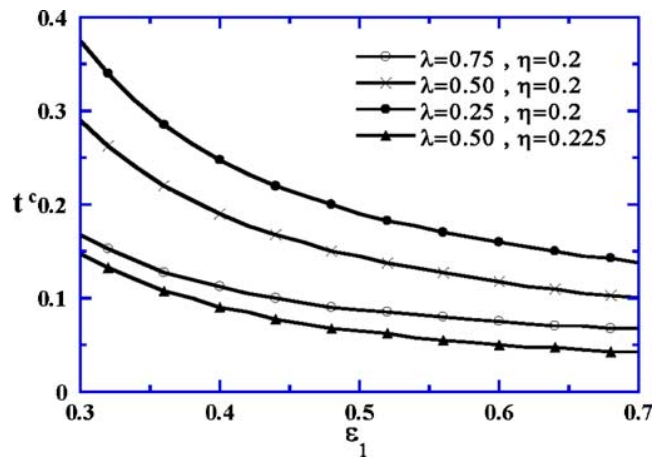

Fig. 10. Effects of GDL porosity $\varepsilon_{1}$ on the time constant. 
system subjected to a start-up operation. In this work, the time constant is defined to be the time needed for the current density reaching $90 \%$ of the value at the corresponding steady state. Fig. 10 presents the effects of GDL porosity, channel width fraction and surface overpotential on the time constant. It is indicated that, in the parameter range considered, the time constant is less than $0.4 \mathrm{~s}$, and increases in GDL porosity, channel width fraction and/or surface overpotential all results in a reduction in time constant.

\section{Concluding remarks}

The dynamic behavior of gas transport and cell performance of PEM fuel cell has been examined by the diffusion transport model developed in the present work. With the assumption of the two-dimensional mass transport in the cathode side of PEMFC, the effects of the channel width fraction, $\lambda=\ell_{\mathrm{c}} / \ell_{\mathrm{b}}$, the porosity of the gas diffuser layer, $\varepsilon_{1}$, and the surface overpotential of the catalyst layer, $\eta$, on the transient characteristics of the resultant current density and mass transport are focused. Based on the results and analysis, the following conclusions can be drawn.

(1) Surface overpotential $\eta$ represents the voltage loss due to chemical reaction within the catalyst layer. A higher surface overpotential means a fuel cell with a faster surface reaction rate. Therefore, a faster response is experienced for the PEM fuel cell when it is started up from the stationary condition.

(2) For a larger channel width fraction, a faster response as well as better cell performance can be attained.

(3) Morphology of the porous layers such as the gas diffusion layer in the present half-cell model is characterized by the porosity. The present results with porosity in the range of 0.3-0.7 show that the full response time is longer for a PEM fuel cell with a higher GDL porosity. While the time constant is shorter for a cell with higher GDL porosity.

(4) It is disclosed that an increase in $\lambda$, or $\eta$ may lead to a faster dynamic response of a PEM fuel cell subjected a started-up operation. Although the dynamic response time may be as long as $10 \mathrm{~s}$ due to the mass transport lag, the fuel cell system needs only about $0.4 \mathrm{~s}$ to reach the $90 \%$ of the current density at the corresponding steady state.

\section{Acknowledgement}

The study was supported by the National Science Council, the Republic of China through the grant numbers NSC 922212-E-002-096, NSC 91-2218-E-035-002, NSC-92-2212E-035-027 and NSC 92-2623-7-002-006-ET.

\section{References}

[1] D.N. Bernadi, M.W. Verbrugge, Mathematical model of a gas diffusion electrode bonded to a polymer electrolyte, AIChE J. 37 (8) (1991) 1151-1163.

[2] G. Xie, T. Okada, Water transport behavior in Nafion 117 membranes, J. Electrochem. Soc. 142 (9) (1995) 3057-3062.

[3] G. Xie, T. Okada, Pumping effects in water movement accompanying cation transport across Nafion 117 membranes, Electrochim. Acta 41 (9) (1996) 1569-1571.

[4] T. Okada, G. Xie, M. Meeg, Simulation for water management in membranes for polymer electrolyte fuel cells, Electrochim. Acta 43 (14-15) (1998) 2141-2155.

[5] T. Okada, G. Xie, O. Gorseth, S. Kjelstrup, N. Nakamura, T. Arimura, Ion and water transport characteristics of Nafion membranes as electrolytes, Electrochim. Acta 43 (1998) 3741-3747.

[6] T. Okada, Theory for water management in membranes for polymer electrolyte fuel cells. Part 1. The effect of impurity ions at the anode side on the membrane performances, J. Electroanal. Chem. 465 (1999) 1-17.

[7] T. Okada, Theory for water management in membranes for polymer electrolyte fuel cells. Part 2. The effect of impurity ions at the cathode side on the membrane performances, J. Electroanal. Chem. 465 (1999) 18-29.

[8] V. Gurau, F. Barbir, H. Liu, An analytical solution of a half-cell model for PEM fuel cells, J. Electrochem. Soc. 147 (12) (2000) 4485-4493.

[9] S. Dutta, S. Shimpalee, J.W.V. Zee, Numerical prediction of massexchange between cathode and anode channels in a PEM fuel cell, Int. J. Heat Mass Transfer 44 (2001) 2029-2042.

[10] J.S. Yi, T.V. Nguyen, Multi-component transport in porous electrodes of proton exchange membrane fuel cells using the interdigitated gas distributors, J. Electrochem. Soc. 146 (1) (1999) 38-45.

[11] W. He, J.S. Yi, T.V. Nguyen, Two-phase flow model of the cathode of PEM fuel cells using interdigitated flow fields, AIChE J. 46 (10) (2000) 2053-2063.

[12] R.J. Kee, P. Korada, K. Walters, M. Pavol, A Generalize model of the flow distribution in channel networks of planar fuel cells, J. Power Sources 109 (2002) 148-159.

[13] E. Hontabon, M.J. Escudero, C. Bautista, P.L. Garcia-Ybarra, L. Daza, Optimisation of flow-field in polymer electrolyte membrane fuel cells using computational fluid dynamics techniques, J. Power Sources 86 (2001) 363-368.

[14] S. Um, C.Y. Wang, K.S. Chen, Computational fluid dynamics modeling of proton exchange membrane fuel cells, J. Eelectrochem. Soc. 147 (12) (2000) 4485-4493.

[15] W.M. Yan, C.Y. Soong, F. Chen, H.S. Chu, Effects of flow distributor geometry and diffusion layer porosity on reactant gas transport and performance of proton exchange membrane fuel cells, J. Power Sources 125 (2004) 27-39.

[16] M. Francesco, E. Arato, Start-up analysis for automotive PEM fuel cell systems, J. Power Sources 108 (2002) 41-52.

[17] S. Yerramalla, A. Davari, A. Feliachi, T. Biswas, Modeling and simulation of the dynamic behavior of a polymer electrolyte fuel cells, J. Power Sources 124 (2003) 104-113.

[18] S. Um, C.Y. Wang, Three-dimensional analysis of transport and electrochemical reactions in polymer electrolyte fuel cells, J. Power Sources 125 (2004) 40-50.

[19] F. Chen, Y.G. Su, C.Y. Soong, W.M. Yan, H.S. Chu, Transient behavior of water transport in the membrane of PEM fuel cell, J. Electroanal. Chem. 566 (2004) 85-93.

[20] S.V. Patankar, Numerical Heat Transfer and Fluid Flow, Hemisphere/McGraw-Hill, New York, 1980, Chapter 6. 